\title{
Art Therapy: Intervention Study of Immersive Interaction Animation on Children with ADHD
}

\author{
Yunpeng Tang ${ }^{1}$ \\ ${ }^{1}$ Academy of Fine Arts, Huanggang Normal University, Huanggang 438000, Hubei, China
}

\begin{abstract}
The prevalence of attention deficit hyperactivity disorder (ADHD) among children and the ineffective traditional treatment methods have become difficult social problems nowadays. From the perspective of art therapy, this paper has put forward the concept of interaction, and considering the clinical experience related to the pathology of children with ADHD, focused on the analysis of the requirements, design and implementation methods of immersive interactive animation in the treatment of $\mathrm{ADHD}$, so as to provide theoretical basis for interdisciplinary research of animation arts in the field of ADHD treatment.
\end{abstract}

\section{Introduction}

Attention deficit hyperactivity disorder (ADHD) is a common psychological disorder of childhood, which seriously endangers the physical and mental health of teenagers. It has received a wide range of attention from the society due to its high incidence among children. The cause of the disease is still unknown, and it is mainly treated with personalized and comprehensive schemes which includes drug therapy, psychotherapy and social rehabilitation training. According to the clinical trials, although drug therapy has certain effect on stabilizing the emotions of Children with ADHD, it will have a negative impact on children's growth and development. Besides, speech as a medium to implement modern psychotherapy cannot solve all psychological problems. It is only effective in correcting irrational cognition and thinking, but helpless in dealing with psychological problems with emotional distress as the main symptom. From the perspective of art therapy, this paper has discussed the demand and design of immersive interactive animation in the treatment of Children with ADHD, and explored and analyzed the theory and practice of interdisciplinary research of animation arts in the field of the treatment of ADHD.

\section{Art Therapy}

Art therapy, also known as art psychotherapy, involves the use of expressive arts such as drawing, music, dance, drama, visual message and poetry to enable patients to have free association in appreciation and practice, and adjust and stabilize their emotions and mood, gradually curing psychological and mental diseases. Art therapy provides opportunities for children's nonverbal expression and communication, and is gradually accepted and applied because it can better reflect the characteristics of children's psychological development, as shown in Figure 1.

\begin{tabular}{|c|c|}
\hline Methods & Characteristics of Art Therapy \\
\hline $\begin{array}{l}\text { (1) Music therapy } \\
\text { (2)Drawing } \\
\text { therapy } \\
\text { (3)Drama therapy } \\
\text { (psychodrama) } \\
\text { (4) Dance therapy } \\
\text { (5)Calligraphy } \\
\text { therapy } \\
\text { (6) Pottery making } \\
\text { (7) Games } \\
\text { (8) Sand tray }\end{array}$ & $\begin{array}{l}\text { (1) Non-verbal communication skills, a wide } \\
\text { range of treatment populations } \\
\text { (2) Strong therapeutic relationship } \\
\text { (3) Most works of art can reveal the contents } \\
\text { of the subconscious } \\
\text { (4) With the conformability of time and } \\
\text { space and the authenticity of this place and } \\
\text { this moment } \\
\text { (5) Promoting the integration of sensory } \\
\text { experience and cognitive thinking } \\
\text { (6)Providing a safe channel to express and } \\
\text { release emotions } \\
\text { (7)Enhancing interaction and cohesion } \\
\text { (8)to cultivate sense of self-identity }\end{array}$ \\
\hline
\end{tabular}

With the development of technologies including the Internet, digital arts, interaction and mobile terminals, technology and art have also been highly integrated. As a result, many arts are presented in more intuitive, three-dimensional and interactive forms, like visualization and interaction of music, digital audio-visual integration system, etc. Interactive animation is a new form of digital animation art, which is gradually formed and developed with the emergence and development of computer multimedia, network technology and interactive technology. Animation player supports incident response, and interactivity is its basic characteristic. By watching such animation, the audience is highly engaged, so as to obtain an immersive experience.

\section{The demand analysis of interactive animation treatment for Children with ADHD}

Symptoms of Children with ADHD include attention deficit, short attention span, hyperactivity and impulsive behavior, 
which are not commensurate with their age and level of development. Although there is no conclusion concerning the causes of disease for Children with ADHD, from the perspective of art therapy, the demands are mainly as follows.

\subsection{Visualization and attention attraction solutions for Children with ADHD}

For a long time, psychological education is mainly carried out by personal example as well as verbal instruction, and Children with ADHD sense and understand the content of psychological education mostly by hearing. However, experiments show that the visual neural system is more sensitive than auditory neural system. For the same information, visual sense can better consolidate information memory. The digital information nowadays has realized the visualization and animation of contents of psychological education which are no longer limited to teaching by sense of hearing, but by the dual stimulation of visual and auditory sense. Thus, it will inevitably be more vivid and can attract more attention of Children with ADHD.

With the application of animation, psychological education is no longer limited to sense of hearing, but uses exquisite pictures, lovely and humorous characters, exaggerated and funny actions, interactive story lines and beautiful music to impress Children with ADHD and attract their attention. When they are absorbed in the plot as characters in animation, with the story of psychological education animation unfolded, it can cultivate and develop their attention, cultivate their habit of starting and ending well, develop their self-control ability and teach them the truth of behaving themselves and dealing with people, the way to get along with each other, and the courage to overcome difficulties by trying to let them to perform their own stories.

\subsection{Interaction and interactive behavior solution for Children with ADHD}

Clinically, it is shown that interactive behavior is critical in the treatment of Children with ADHD. Children with ADHD have symptoms like low concentration and easy to be distracted, so that they need some attractions and good interaction is the key to attraction. Interactive animation supports incident response and interactive functions when being played, and audiences can control and interact with the viewer when watching it. Such control can be some operations of the flash player or operations prepared in advance during the animation production process. Interactivity is the basic feature of interactive animation. When Children with ADHD scream, click, slide, or drag, etc., they can be "understood" by the animation who will then give feedbacks, and thus enhance the desire of Children with ADHD to explore more. The application of this technology enables children with ADHD to get outside of themselves and communicate with the outside world in an unprecedented breadth and depth.

\subsection{Immersion and short attention span solution for children with ADHD}

Children with ADHD are often characterized by symptoms such as short attention span and not long lasting. It is difficult for them to have long-term attention and immersive experience in something. Immersion is a psychological term, which means, when people are involved in a controllable and challenging activity, they will produce a flow, which makes people feel happy and satisfied, and then have an immersion experience. Interactive animation not only is a simple visual presentation of story development, but also fully considers the importance of interactive function. There is an immediate feedback on any interaction, which makes people feel that there is response for any interaction and will make reaction within an acceptable time. Interactive animation allows Children with ADHD to immerse themselves in it instead of participating in it. Finally, the immersive experience leads to the disappearance of our anxiety and the change of our subjective sense of time in activities. At the same time, interactive animation has the characteristics of letting out, sublimation and symbolization, so that children with ADHD can get emotional release and reconciliation.

\subsection{Education and impulsivity solutions for children with ADHD}

The impulsivity of children with ADHD needs to be handled through good psychological counseling and intervention. Animation as an art form based on narration, has important educational functions. While psychological education is visualized, it also integrates its content into the animation story. Children with ADHD are absorbed in the plot when they watch the interactive animation or take themselves as characters in animation. With the story of psychological education animation unfolded, it can cultivate children's habit of starting and ending well, develop their self-control ability and teach them the truth of behaving themselves and dealing with people, the way to get along with each other, and the courage to overcome difficulties by trying to let them to perform their own stories.

\subsection{Mobility and hyperactivity solutions for Children with ADHD}

Children with ADHD have problems such as easy to be distracted and hyperactive. Interactive animation itself has the characteristics of good presentation no matter for people with varied cognitive abilities or in environments of different places regardless of age and environment. In the process of treatment, it has the characteristics of simple operation, easy access and more acceptance. Through the support of mobile terminals, the problem of frequent movement of Children with ADHD can be overcome, so that they can be better applied to the treatment of Children with ADHD.

\section{Design and research of interactive animation in the treatment of children with ADHD}

\subsection{Research on the design of animation visualization to attract children with ADHD}

\subsubsection{Flat design style}

Flat style is adopted in interactive animation modeling. The core meaning of the concept of flat style is to remove 
redundant, heavy and complicated decorative effects, and highlight "information" itself once again. The design elements put emphasis on abstraction, minimalism, symbolization and symbolism. In terms of composition of pictures, it mainly consists of panoramic scene, supplemented by a small number of mid-shot and close-shot scenes and little close-up composition, so as to present an objective narrative angle and reveal the characters and their inner world and emotions through panorama. In terms of modeling, it mostly adopts simple geometric shapes, such as squares, circles, triangles and straight lines. The characters are proportionate, with little or no change in light and shade. What stands out is the proportion and division, and the modeling itself is emphasized.

Compared with the Skeuomorphism, the flat modeling elements have the characteristic of good information transmission. Graphic which is naturally an effective tool to express ourselves is a direct presentation of our subconscious mind, and can directly convey color, movement information and story content to Children with ADHD without extra appreciation and pondering, helping Children with ADHD accept, view and participate in it more intuitively.

\subsubsection{Study of color selection and matching}

The three attributes of color are hue, saturation and value. Different hue can give people different color perception. Colors of high saturation and high purity can better attract children's attention. As Children with ADHD are in their growing stage, they have a high degree of recognition for bright colors, and modeling elements with high saturation and lightness can better attract the attention of Children with ADHD. Therefore, in terms of color design, good chromaticity difference is required to generate rich visual experience, and the tone tends to be vibrant-the color is purer. There are no sharp color gradation or light and shade changes. The color is mainly functional, with gray tone shadows or long shadow, but not blurred. The color edges are clear and neat, the proportion division is reasonable, and there is no redundant decoration. In a word, the screen emphasizes its conciseness, simplicity, as well as the interesting and lively characteristics of animation.

All in all, in terms of the visual design targeted at Children with ADHD, the research focuses on modeling style and color matching. It should be diversified and changeable, simple and great in visual expression. We aim to realize the diversified characteristics of visual elements and create mixed expressions of reality and virtuality, abstract and specific. The diversification of visual elements can further enhance the participation enthusiasm of Children with ADHD, so that they will pay more attention to be involved. After all, colorful visual elements are more interactive and participatory than single visual elements.

\subsection{Research on the Design of Animation Story for Children with ADHD}

\subsubsection{The narrative form of micro-animation}

Facing Children with ADHD with the characteristics such as being hyperactive, easy to be distracted and having difficulty in concentrating for a long time, micro-animation, as a product of new media, has the inherent advantage of being highly integrated with Children with ADHD's current lifestyle, with a wide range of subjects, various content forms, various modes of transmission, short duration and relative ease. Moreover, flat style animation is more suitable for being made into micro-animation. Micro-animation is not played at large screens, and has no complicated and lengthy plot, with simple modeling and background character design. The design endowed with audio-visual changes is a wonderful visual feast, which makes Children with ADHD more acceptable.

\subsubsection{Visual representation of psychological education thought}

How to extract and express psychological education ideas creatively has become the key to interactive animation design. The implantation of psychological education content should closely follow the animation storyline, and the thought of psychological counseling should be integrated into the plot. To arouse the interest of Children with ADHD, animation stories should first consider their spiritual needs. Compared with ordinary patients, Children with ADHD are special in some ways. First of all, as far as their age is concerned, their poor ability to make judgments requires animation design to consider their realities. Secondly, due to the particularity of Children with ADHD, it is crucial for interactive animation to be interesting, participatory and immersive. In particular, the generation of immersion can assist in the treatment of the symptom of attention distraction. When Children with ADHD view, participate in and experience interactive animation, they also get correct psychological counseling.

\subsection{Research on the auditory design of interactive animation suitable for Children with ADHD}

\subsubsection{Study on the selection of music and sound effects for Children with $A D H D$}

Music can not only be pleasing to the eye, but also bring a wonderful hearing enjoyment to everyone. Music can also purify the soul and guide people to have noble ideals. We express our emotions through music, and music releases many of our emotions. Different music brings different psychological feelings to listeners, which is the focus of music psychology research.

Children are naturally interested in sound and can strongly feel the stimulation of music. Music therapy is based on psychotherapy for ADHD patients. With the unique artistry and affinity of music, ADHD patients can calm down and be concentrated in the treatment process. It is also important to choose the appropriate music type for Children with ADHD, and music therapy needs to be adjusted according to the actual conditions. Lively music can let people enjoy harmony and beauty. Such music can make children excited and vigorous and promote their physical and mental health. For popular nursery rhymes such as "There is only a good mother", "Big head son and small head father" and "Little Rabbit", children suffering from attention deficit and hyperactivity can understand them, and their will automatically think about them, which is helpful for the treatment. At the same time, the selected lyrics should be able to be well expressed in action language, thus strengthening the children's expressive ability and coordination. 


\subsubsection{Research on music interactive setting for Children with $A D H D$}

There are mainly two types of music therapy for Children with ADHD. The first type is receptive therapy, that is, in the process of treatment, Children with ADHD can achieve the purpose of treatment by appreciating and listening to music. The other type is interactive therapy, which mainly asks Children with ADHD to perform some recreational activities following the pictures according to the music provided and complete treatment. The latter type is more effective.

Music, as a multimedia, plays a one-to-many transmission role by stimulating the central nervous system of human ears, presenting a variety of information and data, especially the emotional information which is important. Through the focused exploration of emotional expression of music, in the design of music interaction, the original analysis of sound waveform data is used to reflect the character images, plot, action and other attributes, so that users do not feel disgusted and can immerse themselves in it. It is important to give timely feedback of emotions detected. The great potential of music in emotional expression will certainly provide a series of new ideas for the treatment of ADHD.

\subsection{Research on the arrangement of animation interactive activities to impress Children with ADHD}

\subsubsection{Research on the arrangement of interactive activities between Children with ADHD and animation}

Through the actual observation of Children with ADHD, it is found that simple animation works cannot attract the attention of Children with ADHD as originally conceived in the design. Fully considering the characteristics of Children with ADHD, such as low concentration and easy distraction, then the expression element of human-computer interaction is added on the basis of animation to let Children with ADHD participate in rather than passively accept it. In order to express interactive operations in all dimensions, and then attract the attention of Children with ADHD, we design the following interactions. The first one is the interaction between visual elements and audience subjects. Secondly, we design the interaction between visual elements and sound elements. Thirdly, we design the interaction between audience subjects and story content. Fourthly, the interaction between audience subjects and music elements is designed. The last one is the interaction between audience subjects and audience subjects. These interactions can realize multi-dimensional interactive operations of all visible visual elements. This is more complicated and diverse than simply clicking on the screen and the computer making responses. Such multi-dimensional and deeper interactions can better attract the attention of Children with ADHD. Through the design concept of human-computer interaction, Children with ADHD participate interactively. With changeable visual techniques of expression and interactive concept with strong feedback, Children with ADHD are immersed in it.
4.4.2 Research on the arrangement of interactive activities between Children with $A D H D$ and other participants

Children with ADHD not only have defects in physiological performance, but also have some defects in social performance. To make up for their social deficiencies, ADHA children need more opportunities and interactions with others. As an adjuvant therapy for Children with ADHD, interactive animation is not only a visual presentation of story content, but also fully considers the importance of interactions, allowing Children with ADHD to immerse themselves in it instead of participating in it. Therefore, on the basis of human-computer interaction of interactive animation, the expression element of human-to-human interaction is added, which realizes all-round interactions of all participants and can constantly bring surprises to a certain extent, so as to enable Children with ADHD to get outside of themselves and into the crowd, interact with people and cultivate their social personality.

\section{Conclusion:}

This paper looks for designs based on the principle and symptoms of ADHD, finds commonness and deficiency from the current clinical therapies of ADHD, searches for a breakthrough from the current development of new science and technology, and seek inspiration and innovation from other art therapies at present. It explores the visualization and animation of psychological education content, and tries to use immersive interactive animation to intervene Children with ADHD with the process attraction- attentionparticipation-interaction-treatment. It enriches the ideas of therapies for Children with ADHD, provides some reference value for family education, school education and social education of Children with ADHD, and provides some theoretical reference value for the multidisciplinary cross connection.

\section{References}

1. Zhu Zhichao. Analysis of the Differences between Interactive Animation and Traditional Animation. Beauty and Times (Part I). 89-91, 2010.05.

2. Sun Qingyao. On the Significance of Promoting Music Therapy in the Field of Special Education. Northern Music. 2018

3. Zhang Chengyi. Design and Implementation of Music Digital Visualization in the Treatment of Children with ADHD. Beijing University of Technology. 2015. 10.

4. Bao Jin. Research on the Design of Rehabilitation Training Products for Children with ADHD Based on Interaction. MA Thesis of Southwest Jiaotong University. 2017.5. 